\title{
Impacto del efecto de borde sobre biomasa aérea almacenada en un bosque seco tropical en Cundinamarca (Colombia)
}

\author{
Carolina Pachón-Venegas ${ }^{1}$, Yeison Vega-G ${ }^{1}$ y Camila Valdés-Cardona ${ }^{1}$
}

Recibido: 23 mayo 2020 | Aceptado: 10 abril 2021

\section{RESUMEN}

El efecto de borde es una de las consecuencias de la fragmentación a la cual se exponen varios parches de bosque nativo y secundario, este genera cambios en las condiciones microclimáticas y en la estructura del bosque, viéndose reflejado en diferentes factores biológicos como la biomasa aérea. Por lo tanto, se hipotetiza que a medida que se incrementa la distancia desde el borde hacia el interior del bosque, cambian las condiciones microclimáticas, siendo estas más favorables para el almacenamiento de mayor biomasa, aumento de altura y diámetro. Así, se establecieron cuatro transectos de $50 \mathrm{~m}$, donde se ubicaron cada $25 \mathrm{~m}$ parcelas de $25 \mathrm{~m}^{2}$, dando un total de 12 . En cada parcela, se tomaron datos de altura, diámetro a la altura del pecho para todas las plantas leñosas con alturas superiores a $1,30 \mathrm{~m}$ y se registraron datos de humedad relativa y temperatura del aire. Se encontraron diferencias en la biomasa entre las parcelas, atribuible principalmente entre la zona del medio con las zonas de borde y del interior del bosque, contrario a lo reportado en la literatura. Similarmente, hay una segregación de las clases altimétricas y diamétricas entre las zonas, ubicándose los árboles con mayor altura y grosor al interior. No obstante, las variables microclimáticas no reflejaron un efecto sobre los atributos de la vegetación, contrario a lo que se reporta en la literatura. Además, se resalta que la zona media poseía menores valores de biomasa, explicados por factores locales del fragmento.

Palabras clave: altura, diámetro a la altura del pecho (DAP), humedad, temperatura del aire.

'Estudiante de Biología, Facultad de Ciencias, Universidad Nacional de Colombia-Sede Bogotá. Cr. 45 \#26-85, Bogotá, Colombia; cpachonv@unal.edu.co 


\section{ABSTRACT}

The edge effect is one of the consequences of the fragmentation to which various patches of native and secondary forest are exposed, causing changes in the microclimatic conditions and the structure of the forest, which are in turn reflected in different biological variables such as aerial biomass. Therefore, it is hypothesized that as the distance from the edge to the interior of the forest increases, the microclimatic conditions change, becoming more favorable for the storage of greater biomass, as well as increases in tree height and diameter. Thus, four $50 \mathrm{~m}$ transects were established, where $25 \mathrm{~m}^{2}$ plots were located every $25 \mathrm{~m}$. In each plot, data on height, diameter at breast height were taken for all woody plants with heights above $1.30 \mathrm{~m}$ and data on relative humidity and air temperature were recorded. While the interior plot had the highest biomass, as expected, the intermediate zone was found to have the lowest biomass, instead of the pattern of a linear increase in biomass away from the edge described in the literature. The lower than expected biomass of the intermediate zone can be explained by local factors proper to the study area. Similarly, there is a segregation of the height and diameter classes between the zones, with the trees with the greatest height and thickness being located in the forest interior. However, the microclimatic variables were not reflected in the attributes of the vegetation, contrary to what is reported in the literature.

Key words: height, microclimatic conditions, DBH, humidity, air temperature

\section{INTRODUCCIÓN}

La fragmentación consiste en el reemplazo de grandes áreas de bosque nativo por otro tipo de cobertura vegetal dejando parches de bosque aislados (Murcia, 1995). Este proceso expone a los organismos del parche a las condiciones del hábitat contrastante en el ecotono, generando cambios en la abundancia de las especies, en la estructura y en las interacciones comunitarias, lo que se conoce como efecto de borde. Este efecto de borde implica alteraciones microclimáticas que se hacen evidentes en un gradiente perpendicular al borde en la temperatura, humedad, luz $y$ velocidad del viento (Astiani et al., 2018; Murcia, 1995; Zurita et al., 2012; Saeed et al., 2019; Wekesa et al., 2019).
El efecto de borde puede tener también implicaciones sobre la producción de biomasa y, por ende, en la capacidad de almacenamiento de carbono de los bosques, lo que afecta también la provisión de algunos servicios ecosistémicos como la regulación climática por captura de dióxido de carbono (Astiani et al., 2018; Razafindratsima et al., 2017; Saeed et al., 2019; Wekesa et al., 2019). Varios autores han reportado un mayor almacenamiento de carbono en biomasa aérea en el interior de los parches que en el borde y se presume que esto se debe, como se mencionó previamente, a los cambios en la estructura del bosque y composición de especies en el borde (Razafindratsima et al., 2017; Wekesa et al., 2019). Algunos de estos cambios 
están relacionados con modificaciones en la alometría de los árboles como respuesta a las alteraciones microclimáticas, o a rasgos propios de las especies que pueden establecerse en el borde y son resistentes, por ejemplo, a condiciones de alta luminosidad y velocidad de viento (Razafindratsima et al., 2017; Wekesa et al., 2019).

Para la región tropical, dichas afecciones son de particular preocupación, debido a que los bosques tropicales almacenan alrededor del $50 \%$ de carbono en su biomasa aérea, manteniendo el ciclo de este elemento a escalas de paisaje y global, además de proveer hábitats que promueven la conservación de la biodiversidad y el funcionamiento de los ecosistemas (Corona-Núñez et al., 2018; Saeed et al., 2019; Wekesa et al., 2019). Pese a que las investigaciones se han concentrado en los bosques húmedos, se sabe que los bosques secos son reservorios de altas densidades de carbono en vegetación y suelos (Corona-Núñez et al., 2018). En Colombia, dadas las condiciones edafológicas, geomorfológicas y climáticas del bosque seco tropical, gran parte de este ha sido reemplazado por cultivos, pasturas para ganado $y$ asentamientos humanos, quedando remanente el $8 \%$ de la cobertura original en un estado de alta fragmentación y sólo el $4 \%$ correspondiente a bosques maduros (González-M et al., 2018; PortilloQuintero y Sánchez Azofeifa, 2014).

El presente trabajo tuvo como objetivo evaluar el impacto del efecto de borde sobre la biomasa aérea almacenada en un bosque seco tropical en Cundinamarca (Colombia).
Se hipotetiza que la producción de biomasa incrementa conforme se aumenta la distancia desde el borde del parche, debido a que las condiciones microclimáticas como humedad y temperatura son más adversas en el borde, generando árboles con menor DAP y altura.

\section{MATERIALES Y MÉTODOS}

\section{Área de estudio}

La Reserva Natural de la Sociedad Civil / VI área protegida con recursos administrados Maná Dulce está ubicada en los municipios de Nilo y Agua de Dios (4²1'03.4"N; 74³9'05.4"W; Fig. $1)$, en el suroccidente del departamento de Cundinamarca (Colombia) (MADS, 2018). Su área comprende 71,0590 hectáreas de bosque seco tropical del valle interandino del río Magdalena, con predominancia de plantas leñosas de hoja ancha, con una altitud media de 450 msnm (MADS, 2018; Martín-Reyes, 2019). Se encuentra en una zona con clima cálido seco, con temperatura promedio de $26^{\circ} \mathrm{C}$, precipitación anual entre 1000 y $1500 \mathrm{~mm}^{3}$ repartida en un régimen bimodal, siendo los meses de mayo y septiembre los más lluviosos, y una humedad relativa media de $70 \%$ (Martín-Reyes, 2019). Las actividades y usos a los que está dedicada la Reserva Maná Dulce son de conservación, preservación, regeneración y restauración de los ecosistemas, incluyendo su fauna y flora. Similarmente, el aprovechamiento sostenible de productos maderables, no maderables, educación ambiental, ecoturismo, la investigación básica, aplicada, como también la generación de bienes y servicios ambientales hacen parte de la gama de usos a los que está destinada la reserva (MADS, 2018). 
El muestreo se llevó a cabo entre el 11 y 13 de noviembre de 2019.

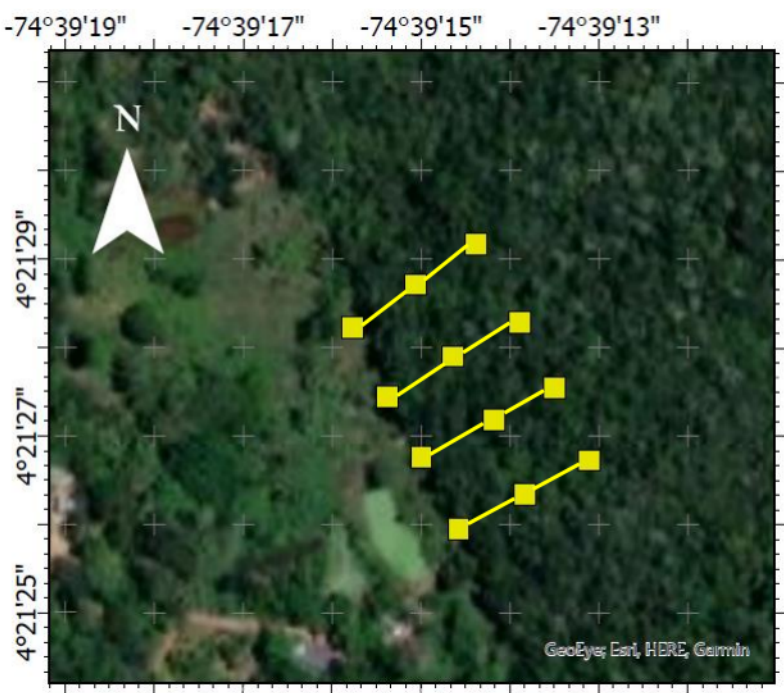

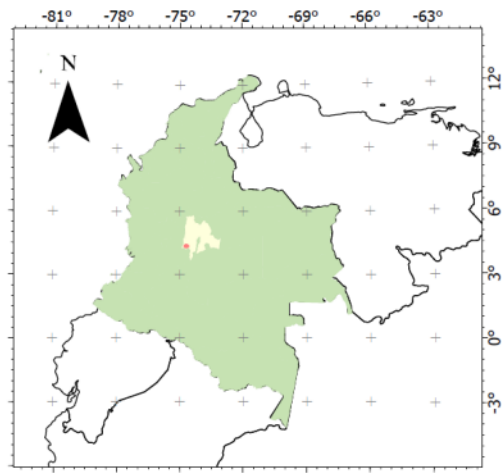

Cundinamarca

Agua de dios

Parcelas

Figura 1.

Mapa de ubicación de los sitios de muestreo, Reserva Maná Dulce, Agua de dios, Cundinamarca (Colombia).

Diseño de muestreo y recolección de datos de vegetación

Se seleccionó aleatoriamente un parche de bosque sobre el cual se trazaron cuatro transectos perpendiculares al borde (ecotono con pastizal para pastoreo) de $50 \mathrm{~m}$ de longitud. En cada transecto se establecieron tres parcelas de $25 \mathrm{~m}^{2}$, separadas $25 \mathrm{~m}$ entre ellas (Fig. 1). Para cada una de las parcelas se midió la altura y diámetro a la altura del pecho (DAP) de todos los individuos que presentaron más de $1,30 \mathrm{~m}$ de altura. Estas medidas fueron tomadas con un clinómetro para calcular la altura y con una cinta métrica para la circunferencia a la altura del pecho; con este dato se calculó posteriormente el DAP.

En cada parcela se tomaron datos de humedad relativa y temperatura del aire con un higrómetro SILVA ADC Pro en un período de tiempo menor a 30 minutos para evitar un sesgo en dichos datos por la hora del día en la que fueran tomados. Estas mediciones fueron registradas antes del mediodía y fueron tomadas en el centro de cada parcela.

\section{Cálculo de biomasa aérea}

Se utilizó la ecuación propuestas por Yepes et al (2011), para calcular la biomasa aérea únicamente con el valor del DAP.

$$
\begin{aligned}
& \ln [B A]=a+B 1 \ln [D] \\
& \text { Yepes et al (2011) }
\end{aligned}
$$

Donde, BA es biomasa aérea $(\mathrm{kg})$, a $(-2,235)$ y $B 1 \quad(2,37)$ constantes correspondientes al bosque seco tropical y D es DAP (cm). Además, 
se estimó la biomasa por cuadrante por medio de la ecuación descrita por Yepes et al (2011).

$$
B A(t / h a)=B A(\mathrm{~kg} / \mathrm{par}) x(1 \mathrm{t} / 1000 \mathrm{~kg}) \times F C
$$

Donde, BA es biomasa aérea y $\mathrm{FC}$ es el factor de conversión que se debe emplear según el tamaño de la parcela utilizado. En este caso, para una parcela de $5 \times 5 \mathrm{~m}$ corresponde al valor de 200 .

\section{Análisis de datos}

Para evaluar si existen diferencias significativas en la biomasa acumulada ocasionada por el efecto de borde, se realizó una prueba de Kruskal-Wallis entre las diferentes parcelas. Para determinar qué parcelas presentaron diferencias significativas en su biomasa almacenada, se realizó un análisis de comparación múltiple post hoc de Dunn aplicando la corrección de Bonferroni.
Respecto a la influencia de las variables microclimáticas (humedad y temperatura) sobre la biomasa, el DAP y la altura, se realizó un Modelo Lineal Generalizado (GLM), debido a la poca colección de variables microclimáticas y a la no normalidad de los datos. El tratamiento de datos fue realizado por medio de los programas Past 3 (Hammer et al, 2001) y Excel $₫$.

\section{RESULTADOS}

Las diferentes variables ambientales y biológicas medidas (Tabla 1), mostraron una alta desviación estándar o alta dispersión de datos. Se destacan los datos de la parte media del bosque, siendo estos los más bajos de los tres puntos en todas las variables. Además, el valor de biomasa de la parte interior del bosque presenta una alta desviación estándar, indicando una alta variabilidad en los tamaños de los árboles medidos en esta zona.

\begin{tabular}{lcccc}
\hline \multirow{2}{*}{ Variables } & \multicolumn{3}{c}{ Promedio (desviación estándar) } \\
\cline { 2 - 5 } & Borde & Medio & Interior & p-value \\
\hline Temperatura $\left({ }^{\circ} \mathbf{C}\right)$ & $23,875( \pm 0,340)$ & $23,800( \pm 0,230)$ & $23,900( \pm 0,244)$ & 0,68 \\
Humedad $(\%)$ & $87,225( \pm 2,134)$ & $88,000( \pm 1,756)$ & $89,000( \pm 1,587)$ & 0,47 \\
Biomasa (t/ha) & $30,417( \pm 19,719)$ & $10,066( \pm 5,221)$ & $52,873( \pm 52,348)$ & $0,016^{*}$ \\
DAP (cm) & $5,017( \pm 3,832)$ & $3,829( \pm 2,103)$ & $5,743( \pm 4,652)$ & $0,016^{\star}$ \\
Altura (m) & $4,611( \pm 1,919)$ & $4,320( \pm 1,287)$ & $5,685( \pm 1,914)$ & $1,71^{*} 10^{-5 * *}$ \\
\hline
\end{tabular}

Nota: La biomasa reportada corresponde a los valores calculados por el método de Yepes et al (2011). * Diferencias significativas según la prueba Kruskal-Wallis con $a=0,05 ;{ }^{* *} \alpha=0,01$.

Tabla 1.

Valores promedio y desviación estándar de todas las variables medidas y obtenidos, según su ubicación en un bosque seco tropical, Reserva Maná Dulce, Colombia. 
La biomasa calculada según la ecuación de Yepes et al. (2011) osciló entre 3,57 t/ha y 127,60 t/ha. Se presentaron diferencias significativas en el cálculo de biomasa almacenada (Kruskal Wallis: $\left.\mathrm{H}\left(\mathrm{chi}^{2}\right)=8,303 ; \mathrm{p}=0,016\right)$, donde el análisis de comparación múltiple post hoc de Dunn indica que las diferencias en el aporte de biomasa se encuentran entre la zona media y del interior del bosque (MedioInterior: $p$-value $=0,003$ ), siendo el interior del bosque la zona que presenta un mayor valor. Por otro lado, las zonas de borde no presentan diferencias significativas con respecto a las zonas medias y del interior del bosque (Borde-Medio: $\mathrm{p}$ value $=0,28$; Borde-Interior: $p$ value $=0,12$; Fig. 2).

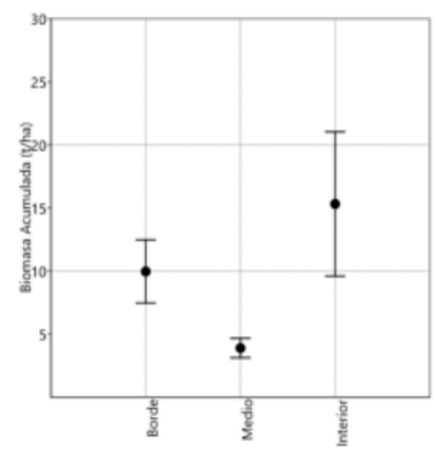

A)

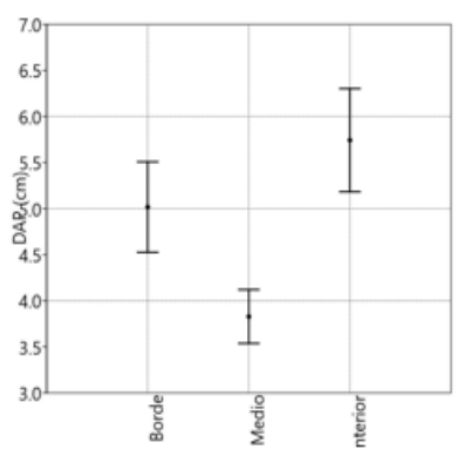

B)

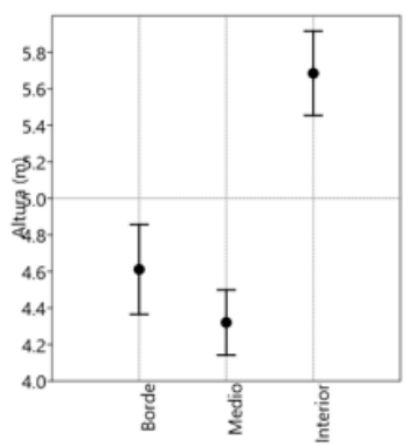

C)

Figura 2.

Boxplot de (a) biomasa acumulada, (b) DAP y (c) altura de los árboles medidos en un bosque seco tropical, Reserva Maná Dulce, Colombia. Los datos se encuentran clasificados según su ubicación en el bosque: en el borde $(0 \mathrm{~m})$, en el medio (25m) $y$ en el interior (50m).

Este patrón de la biomasa se evidencia en la estructura diamétrica, donde la distribución del DAP presentó diferencias significativas entre las zonas del bosque (Tabla 1), especialmente entre las zonas media y del interior (Medio-Interior: $p$-value $=0,013$ ). Así mismo, se evidenció en las clases diamétricas (Fig. 3A) que los árboles con mayores DAP (valores superiores a $7,4 \mathrm{~cm}$ ) son poco frecuentes en las zonas de borde y media del transecto. Por otro lado, a pesar de que la altura presentó diferencias entre las zonas (Tabla 1), no se siguió el mismo patrón descrito anteriormente, en cambio se observó que el interior del bosque es significativamente diferente de las otras dos zonas del transecto (BordeInterior: $\quad \mathrm{p}$-value $=7,5^{*} 10^{-4}$; MedioInterior: p-value $=6,24^{*} 10^{-5}$; Fig. 4C). Al analizar las clases altimétricas (Fig. 3B) evidentemente los árboles más altos tienden a agruparse en las zonas del interior del bosque. 

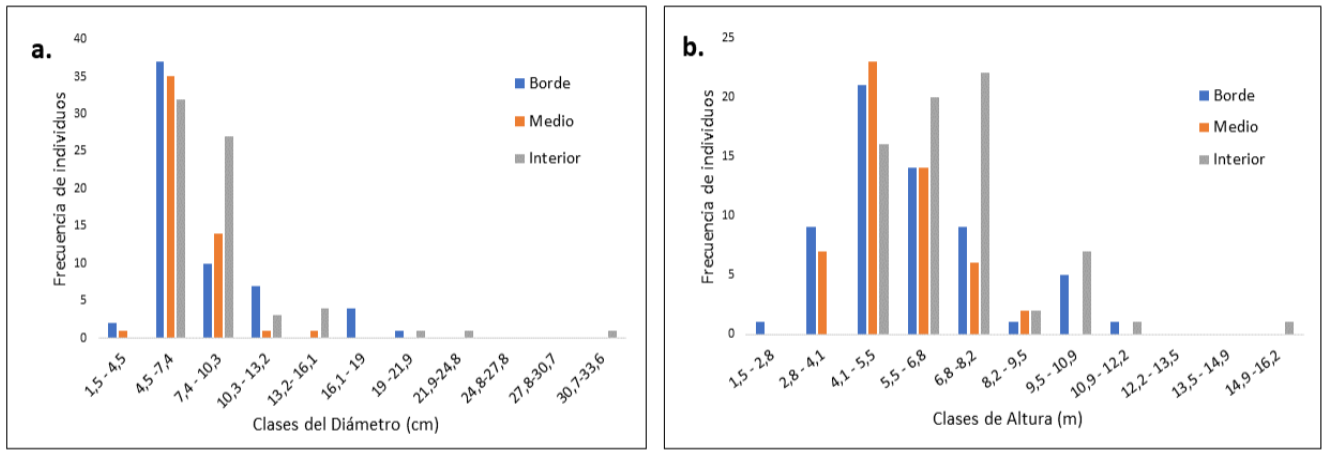

Figura 3.

Clases diamétricas (a) y altimétricas (b) de los árboles medidos en un bosque seco tropical, Reserva Maná Dulce, Colombia. Los datos se encuentran clasificados según su ubicación en el bosque: en el borde $(0 \mathrm{~m})$, en el medio $(25 \mathrm{~m})$ y en el interior (50m); y a la clase en la que se agrupan, siendo 10 clases en total.
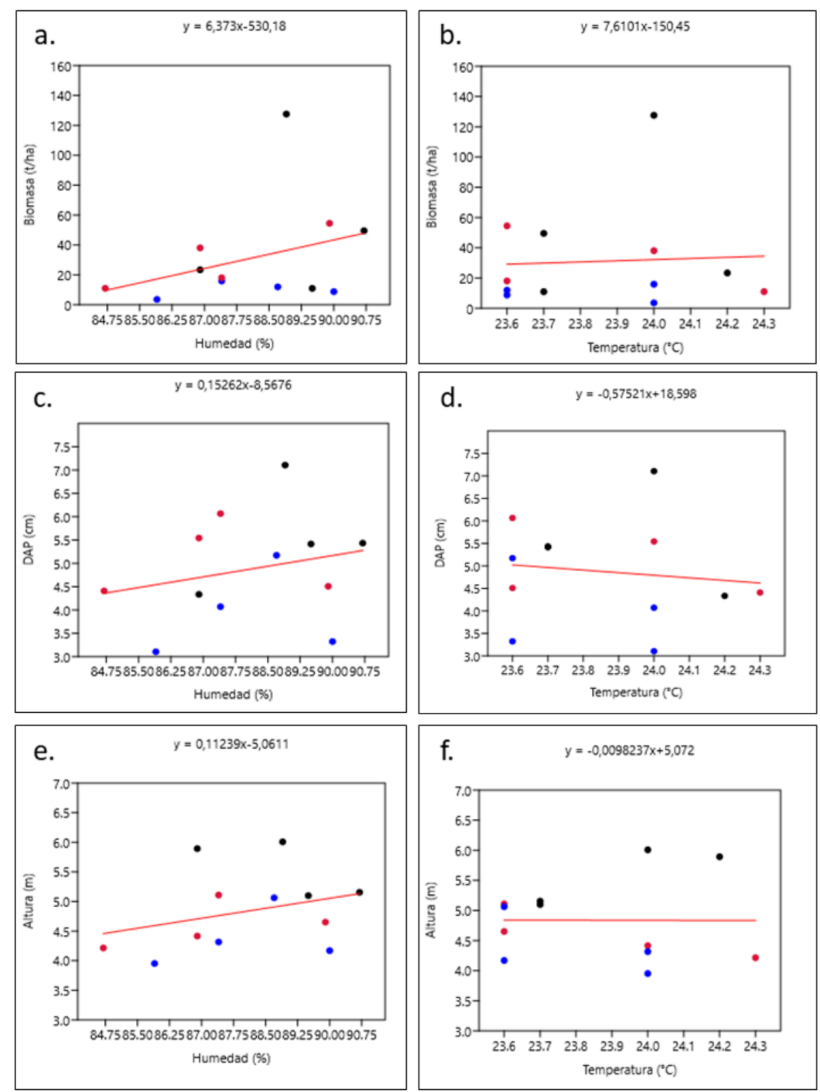

Figura 4.

Modelo Lineal Generalizado (GLM) para las diferentes variables microclimáticas versus las variables biológicas medidas. Los puntos rojos corresponden a los valores del borde del bosque, los azules a la parte media y los negros a la parte interna. Valores $R^{2}$ para cada GLM: a. 0,114; b. 0,003; c. 0,059; d. 0,016; e. 0,095; f. 0,00001. 
Respecto a las variables microclimáticas y su relación con las variables biológicas (Fig. 4) se obtuvo, que solo la humedad posee una relación positiva sobre estas sin importar la ubicación en el bosque, ya que los valores en los diferentes puntos se mantuvieron cercanos 0 casi iguales entre sí. Respecto a la temperatura, no se observa una relación clara con las variables y al igual que con la humedad, no hay cambios de ésta entre los puntos muestreados.

\section{DISCUSIÓN}

El bosque seco tropical de la Reserva Maná Dulce no posee diferencias en la biomasa de los individuos cercanos al borde con respecto a los que se encuentran en la parte interna, es decir, no se evidencia un efecto de este sobre el almacenamiento de carbono, contrario a lo reportado por Magnago et al. (2017), donde sí hay un efecto del borde del bosque sobre la biomasa. Adicionalmente, no se demostró una variación de los factores microclimáticos medidos, lo cual no concuerda con lo reportado en la bibliografía, donde las variables microclimáticas de temperatura y humedad son más adversas en el borde (Bernaschini et al., 2019).

Con respecto a la altura y el DAP, Reinmann y Hutyra (2016) reportan que el crecimiento de los árboles ubicados cerca al borde es tres veces menor con respecto a los árboles del interior del bosque, hecho que se refleja parcialmente en la Reserva Maná Dulce donde los árboles más altos tienden a ubicarse en la zona más lejana al borde (Fig. 3B). No obstante, se encontró que esta segregación altimétrica en la reserva, no responde a la misma causa reportada por Reinmann y Hutyra (2016), los cuales enfatizan que la temperatura es el principal agente que limita el crecimiento en los bordes debido a que causan estrés en el período de crecimiento. De esta manera, se propone que esta diferenciación en las clases altimétricas en la reserva responde a variables ambientales asociadas a la humedad, la cantidad de agua, propiedad del suelo para retener agua, topografía del terreno, entre otras; como se ha reportado para los bosques secos alrededor del mundo (Allen et al., 2016; Chaturvedi y Raghubanshi, 2018; Pizano y García, 2014; Sánchez-Azofeifa et al., 2016).

De forma similar, las clases diamétricas reflejan que los árboles con mayores DAP solo se ubican al interior del bosque, hecho que concuerda con lo reportado por Harper et al (2005) y Astiani et al (2018), donde se describe una relación negativa entre el DAP y la distancia desde el borde; es decir, en los bordes los DAP decrecen significativamente y estos restringen el crecimiento de biomasa aérea por unidad de área.

Cabe resaltar, los datos de la parte media del bosque presentaron diferencias con las otras dos zonas, siendo estos los más bajos en todas las categorías. Aunque no es clara la razón por las variables medidas, se hipotetiza que estas variaciones en la biomasa y, consecuentemente, en el DAP son causadas por factores locales de ese fragmento, como la topografía y la edad del bosque (Fagan et al., 2016; Fonseca et al., 2011; Lertzman et al., 1996; Thomas et al., 1979). 
Por lo tanto, se recomienda que las principales variables microclimáticas a tener en cuenta en un estudio de influencia del efecto de borde sean: la temperatura, la humedad, la velocidad del viento; incluyendo otras como la topografía, el tiempo de desarrollo del borde y la densidad del follaje (Davies-Colley et al., 2000; Razafindratsima et al., 2017). Además, la influencia del efecto de borde es menor a medida que madura el borde del bosque, en bosques húmedos tropicales la influencia ocurre en los primeros $40 \mathrm{~m}$ desde el borde y va decreciendo a medida que el bosque madura (Davies-Colley et al., 2000; Wang et al., 2019).

\section{CONCLUSIONES}

En conclusión, la hipótesis principal es descartada, contrario a lo reportado en otros estudios. De esta manera, es necesario mejorar los métodos de toma de datos microclimáticos, aumentar las variables y el tamaño del transecto (Magnago et al., 2015). Por otro lado, se demostraron algunas de las predicciones hechas; la altura sí aumenta a lo largo del bosque, es decir desde el borde hacia el interior, y el DAP exhibe una relación inversa a medida que aumenta la distancia desde el borde. Además, se reporta que los datos de la parte media del bosque son más bajos que los de las otras dos zonas, lo que plantea nuevas hipótesis para futuros estudios a realizar en la zona.

\section{AGRADECIMIENTOS}

A la Universidad Nacional de Colombia y al Departamento de
Biología por permitir la realización de la salida de campo y abrir espacios para la investigación de los bosques del país. A la profesora Beatriz Salgado por la corrección del manuscrito, su apoyo en campo $y$ en las sugerencias para el presente estudio. A Thibaud Aronson por su colaboración en correcciones de estilo y su lectura crítica. Agradecimientos especiales a la Dra. Andrea Martínez por su apoyo continuo.

\section{BIBLIOGRAFÍA}

Allen, K., Dupuy, J.M., Gei, M., Hulshof, C., Medvigy, D., Pizano, C., et al. (2017). Will seasonally dry tropical forests be sensitive or resistant to future changes in rainfall regimes? Environmental Research Letters, 12, 1-15. https:// doi.org/10.1088/1748-9326/aa5968.

Astiani, D., Curran, L.M., Mujiman, Ratnasari, D., Salim, R., Lisnawati, N. (2018). Edge effects on biomass, growth, and tree diversity of a degraded peatland in West Kalimantan, Indonesia. Biodiversitas, 19, 272-278. https:// doi.org/10.13057/biodiv/d190137

Bernaschini, M.L., Trumper, E., Valladares, G., Salvo, A. (2019). Are all edges equal? Microclimatic conditions, geographical orientation and biological implications in a fragmented forest. Agriculture, Ecosystems \& Environment, 280, 142-151. https://doi.org/10.1016/ j.agee.2019.04.035 
Chave, J., Muller-Landau, H.C., Baker, T.R., Easdale, T.A., ter Steege, H., Webb, C.O. (2006). Regional and phylogenetic variation of wood density across 2456 neotropical tree species. Ecological Applications, 16(6), 2356-2367. https://doi.org/10.1890/1051-0761

Corona-Núñez, R.O., Campo, J., Williams, M. (2018). Aboveground carbon storage in tropical dry forest plots in Oaxaca, Mexico. Forest Ecology and Management, 409, 202214. https://doi.org/10.1016/ j.foreco.2017.11.014

Davies-Colley, R.J., Payne, G.W., Van Elswijk, M. (2000). Microclimate gradients across a forest edge. New Zealand Journal of Ecology, 24(2):111-121.

Fagan, M.E., Morton, D.C., Cook, B., Masek, J.G., Zhao, F.A., Nelson, R., Huang, C. (2016). Beyond edge effects: Landscape controls on forest structure in the southeastern US: AGU Fall Meeting Abstracts.

Fonseca, W., Rey-Benayas, J.M., Alice, F.E. (2011). Carbon accumulation in the biomass and soil of different aged secondary forests in the humid tropics of Costa Rica. Forest Ecology and Management, 262(8), 1400-1408. https:// doi.org/10.1016/j.foreco.2011.06.036
González, M. R., García, H., Isaacs, P., Cuadros, H., López-Camacho, R., Rodríguez, N., Pérez, K., Mijares, F., Castaño-Naranjo, A., Jurado, R., Idárraga-Piedrahita, A., Rojas, A., Vergara, H., Pizano, C. (2018). Disentangling the environmental heterogeneity, floristic distinctiveness and current threats of tropical dry forest in Colombia. Environmental Research Letters, 13: 045007. https: / / doi.org/10.1088/1748-9326/aaad74

Hammer, Ø., Harper, D.A.T., Ryan, P.D. (2001). PAST: Paleontological statistics software package for education and data analysis. Palaeontologia Electronica, 4(1): 9pp. http: //palaeoelectronica.org/2001_1/past/

issue1_01.htm

Harper, K., MacDonald, E., Burton, P.J., Chen, J., Brosofske, K.D. (2005). Edge Influence on Forest Structure and Composition in Fragmented Landscapes. Conservation Biology, 19 (3): 769-782. https://doi.org/10.1111/ j.1523-1739.2005.00045.x

Letzman, K.P., Sutherland, G., Inselberg, A., Saunders, S.C. (1996). Canopy gaps and the landscape mosaic in a temperate rainforest. Ecology, 77: 1254-1270. https:// doi.org/10.2307/2265594 
Magnago, L.F.S., Magrach, A., Barlow, J., Schaefer, C.E.G.R., Laurance, W.F., Martins, S.V., Edwards, D.P. (2017). Do fragment size and edge effects predict carbon stocks in trees and lianas in tropical forests? Functional Ecology, 31(2), 542-552. https://

doi.org/10.1111/1365-2435.12752

Magnago, L.F.S., Rocha, M.F., Meyer, L., Martins, S.V., Meira-Neto, J.A.A. (2015). Microclimatic conditions at forest edges have significant impacts on vegetation structure in large Atlantic forest fragments. Biodiversity and Conservation, 24(9), 2305-2318. https://doi.org/10.1007/ s10531-015-0961-1

Martín-Reyes, J.C. (2019). Estudio de caso en la Reserva Mana Dulce: Construcción e implementación de la cartilla En las alas del murciélago. En: Martín-Reyes, J.C. De mitos a verdades: Una cartilla de divulgación científica acerca de la importancia ecológica de los murciélagos. Tesis de maestría. Facultad de Ciencias, Universidad Nacional de Colombia.

Ministerio de Medio Ambiente y Desarrollo Sostenible (MADS). (2018). Resolución número 037 del 23 de marzo de 2018. p. 19.

Murcia, C. (1995). Edge effects in fragmented forests: implications for conservation. Trends Ecology Evolution, 10(2): 58-62. https:// doi.org/10.1016/ s0169-5347(00)88977-6

Pizano, C., García, H. (2014). El Bosque seco Tropical en Colombia. Instituto de Investigación de Recursos Biológicos Alexander von Humbolt. Bogotá, D.C. Colombia. p. 228.
Portillo-Quintero, C.A., SánchezAzofeifa, G.A. (2010). Extent and conservation of tropical dry forests in the Americas. Biological Conservation, 143(1): 144-155. https://doi.org/10.1016/

j.biocon.2009.09.020

Razafindratsima, O.H., Brown, K.A., Carvalho, F., Johnson, S.E., Wright, P.C., Dunham, A.E. (2017). Edge effects on components of diversity and above-ground biomass in a tropical rainforest. Journal of Applied Ecology, 55(2): 977-985. https://

doi.org/10.1111/1365-2664.12985

Reinmann, A.B., Hutyra, R. (2017). Edge effects enhance carbon uptake and its vulnerability to climate change in temperate broadleaf forests. Proceedings of the National Academy of Sciences, 114: 1-6. https://doi.org/10.1073/

pnas. 1612369114.

Saeed, S., Yujun, S., Beckline, M., Chen, L., Zhang, B., Ahmad, A., Mannan, A., Khan, A., Iqbal, A. (2019). Forest edge effect on biomass carbon along altitudinal gradients in Chinese Fir (Cunninghamia lanceolata): A study from Southeastern China. Carbon Management, 10(1): 1-12. https:// doi.org/10.1080/17583004.2018.153 7517

Sánchez-Azofeifa, A., Powers, J., Fernandes, G., Quesada, M. (2014). Tropical dry forests in the Americas: Ecology, Conservation and Management. Taylor and Francis Group. pp 17; 35. 
Thomas, J.W., Maser, C., Rodiek, J.E. Edges. In: Thomas, J.W. editor. (1979). Wildlife habitats in managed forests: The Blue Mountains of Oregon and Washington. U.S. Department of Agriculture Forest Service, p. 48-59.

Wang, G., Guan, D., Xiao, L., Peart, M.R. (2019). Forest biomass-carbon variation affected by the climatic and topographic factors in Pearl River Delta, South China. Journal of Environmental Management, 232, 781788. https://doi.org/10.1016/ j.jenvman.2018.11.130

Wekesa, C., Kirui, B.K., Maranga, E.K., Muturi, G.M. (2017). Variations in forest structure, tree species diversity and above-ground biomass in edges to interior cores of fragmented forest patches of Taita Hills, Kenya. Forest Ecology and Management, 440:48-60. https: / / doi.org/10.1016/

j.foreco.2019.03.011.

Yepes, A.P., Navarrete, D.A., Duque, A.J., Phillips, J.F., Cabrera, K.R., Álvarez, E., García, M.C., Ordoñez, M.F. (2011). Protocolo para la estimación nacional y subnacional de biomasa -carbono en Colombia. Instituto de Hidrología, Meteorología, y Estudios Ambientales-IDEAM. pp. 162.

Zianis, D. (2008). Predicting mean aboveground forest biomass and its associated variance. Forest Ecology and Management, 256: 1400-1407. https://doi.org/10.1016/

j.foreco.2008.07.002.
Zurita, G., Pe'er, G., Bello, M.I., Hansbauer, M.M. (2012). Edge effects and their influence on habitat suitability calculations: a continuous approach applied to birds of the Atlantic forest. Journal of Applied Ecology, 49(2): 503-512. https:// doi.org/10.1111/ j.1365-2664.2011.02104.x 